Conclusion This study demonstrates that social disadvantage as measured by low SEP is correlated with increased HPA axis activity. The conceptualisation of social disadvantage as a chronic stressor may be valid and previous studies reporting no associations between SEP and hair cortisol may have some methodological limitations. Future analyses using biosocial data may need to take into account and adjust for missing data in biosocial analyses.

\section{OP85 INVESTIGATING THE HEALTH OF OLDER CARERS WITHIN DIFFERENT SOCIAL CARE SYSTEMS IN IRELAND AND ENGLAND: EVIDENCE FROM THE IRISH LONGITUDINAL STUDY ON AGEING (TILDA) AND THE ENGLISH LONGITUDINAL STUDY OF AGEING (ELSA)}

${ }^{1}$ CA McGarrigle*, 'S Leahy, ${ }^{1,2}$ RA Kenny. 'The Irish Longitudinal Study on Ageing, Trinity College Dublin, Dublin, Ireland; '2Mercer's Institute for Successful Ageing, St James' Hospital, Dublin, Ireland

\subsection{6/jech-2018-SSMabstracts.84}

Background The continuing social participation of older people through informal caring of family members and friends provides an important economic and social contribution to society. Participation in leisure activities and supportive social ties have been associated with improved physical and mental health. These health effects work through a number of pathways, both behavioural and psychological, which can result in better health behaviours, and buffering of the harmful effects of stress. We investigated and compared the prevalence of the provision of informal caring by the older population in Ireland and England. We investigated predictors of, and health outcomes associated with informal caring and determined whether these are moderated by social participation and receipt of formal care services and support in two different social care systems.

Methods We examined measures of physical function (handgrip strength), mental health (CES-D, CASP-12), economic and social participation, associated with provision of informal care, using multivariate models, in the Irish Longitudinal Study on Ageing (TILDA) (Wave 3, 2014, $n=6,649$ ), and the English Longitudinal Study of Ageing (ELSA) (Wave 6, 2012-2013, $\mathrm{n}=10,372$ ). Caring was defined as having provided care for at least one hour: in the last month, the last week and the numbers of hours of care.

Results A higher proportion of the population aged 50 and over reported caring in the last month in England (22.3\%) compared to Ireland (8.9\%). Caring was associated with similar characteristics (younger age, female, not employed and being married). There were similar health outcomes for both countries. The health outcomes associated with providing low intensity caring ( $<20$ hours/week) were higher grip strength (ELSA $\beta=1.12(95 \%$ CI $0.63,1.61) \mathrm{p}<0.001)$, higher quality of life (TILDA $\beta=1.23$ (95\% CI 0.44, 2.03) $\mathrm{p}=0.002$; ELSA $\beta=0.38(95 \%$ CI $-0.01,0.78) p=0.056)$ and lower depressive symptoms (ELSA $\beta=-0.11$ (95\% CI $-20,-0.03) \mathrm{p}=0.007)$. In contrast, intensive provision of care $(50+$ hours/week), was associated with lower quality of life (ELSA $\beta=-1.57$ (95\% CI $-2.19,-0.95), \mathrm{p}<0.001)$ and more depressive symptoms (TILDA $\mathrm{B}=1.02 \quad(95 \% \quad 0.24,1.80) \quad \mathrm{p}=0.01 ; \quad$ ELSA $\mathrm{B}=0.27$ (95\% CI $0.14,0.41) \mathrm{p}<0.001)$. These associations were moderated by active social activities, positive social relationships and access to formal care services $(p=0.05)$.
Conclusion Across two separate social care systems, the older population contribute substantially to the support and informal care of their family and friends. Overall, informal caring was associated with positive health outcomes, but this depended on both intensity of care provided and accessibility to both social supports and formal care provision.

\section{Late breaking abstracts}

\section{LB1 IS HIGHER PERCEIVED SOCIAL SUPPORT RELATED TO GREATER PARTICIPATION IN CARDIOVASCULAR DISEASE (CVD) RISK REDUCING BEHAVIOURS FOR PEOPLE WITH SEVERE MENTAL ILLNESSES (SMI)?}

${ }^{1} \mathrm{~A}$ Burton*, ${ }^{2} \mathrm{~K}$ Walters, ${ }^{1} \mathrm{D}$ Osborn. 'Division of Psychiatry, University College London, London, UK; ${ }^{2}$ Department of Population Health and Primary Care, University College London, London, UK

\subsection{6/jech-2018-SSMabstracts.85}

Background The mortality gap for people with SMI from CVD is increasing. Studies have found a positive relationship between perceived social support and CVD outcomes in the general population; however there has been less research in people with SMI. Social support may influence CVD outcomes through encouraging participation in healthy lifestyle activities. Aims To assess whether higher social support as measured by the Medical Outcomes Study-Social Support Survey (MOSSSS) is associated with greater attendance at primary care intervention appointments. Secondary outcomes included:

- adherence to CVD medications (Morisky Medication Adherence Scale (MMAS)),

- physical activity (International Physical Activity Questionnaire (IPAQ)),

- $\operatorname{diet}$ (Dietary Instrument for Nutrition Education (DINE))

- alcohol consumption (Alcohol Use Disorders Identification Test (AUDIT)),

- self-reported smoking behaviour

Methods Longitudinal and cross sectional studies involving 326 patients with SMI and raised CVD risk factors. Participants were recruited from $76 \mathrm{GP}$ practices in England to a clinical trial assessing the effectiveness of a behavioural intervention for reducing CVD risk in people with SMI against routine GP practice care. Secondary data analysis used random effects linear regression models for continuous outcomes, logistic regression for binary outcomes, and Poisson/negative binomial regression models for count outcomes.

Results A weak association between social support and attendance at primary care intervention appointments was identified. As social support scores increased by one point, the appointment attendance rate increased by $0.5 \% \quad(\mathrm{IRR}=1.0053$; 1.0001-1.0105, $\mathrm{p}=0.05$ ). When sex, age, ethnicity, diagnosis and deprivation were entered into the model, this association decreased to $0.3 \%$ and was no longer significant $(\mathrm{IRR}=1.0032 ; 0.9980-1.0085, \mathrm{p}=0.23)$.

A strong association between social support and CVD medication adherence was identified. The odds of being in the moderate/high adherence group compared to the low adherence group increased by $3.9 \%$ with a one point increase on the MOS-SSS $(\mathrm{OR}=1.0385 ; 1.0176$ to $1.0598, \mathrm{p}<0.001)$. This association remained significant when sex, age, ethnicity, 